Dept. of Math./CMA University of Oslo

PuRE Mathematics No 18

ISSN $0806-2439$

August 2009

\title{
Optimal stopping of stochastic differential equations with delay driven by Lévy noise
}

\author{
Salvatore Federico ${ }^{1} \quad$ Bernt $\varnothing_{k s e n d a l}^{2,3}$
}

4 August 2009

\begin{abstract}
We study an optimal stopping problem for a stochastic differential equation with delay driven by a Lévy noise. Approaching the problem by its infinite-dimensional representation, we derive conditions yielding an explicit solution to the problem. Applications to the American put option problem are shown.
\end{abstract}

Keywords: Optimal stopping, stochastic delay equations, linear evolution equation, free boundary problem.

A.M.S. Subject Classification: 60G40, 34K50, 47D06.

\section{Introduction}

This paper is involved with the problem of optimal stopping of a linear stochastic differential equation with delay driven by a Lévy noise.

The problem of optimal stopping of stochastic systems is a very important topic in the field of stochastic control theory. From the theoretical point of view, it presents a very deep connection with free-boundary problems. From the applied point of view, historically, it was strictly connected with the problem of the optimal exercise time for the American option. We refer to the monograph [18] for a survey on this topic in a very general setting.

To our knowledge, the only paper dealing with the problem of optimal stopping of delay systems is [9]. However, contrary to our case, it works only with a system driven just by a Brownian noise.

Delay systems are quite hard to study due to their lack of markovianity. In particular, the Dynamic Programming Theory cannot be applied, as it is, for the study of control problems dealing with delay systems. Basically, the difficulty arises from the fact that delay systems have, in general, an infinite-dimensional nature. Hence, the only general approach to them has to be infinite-dimensional. The idea behind this kind of approach is quite simple: instead to consider as state variable only the present of the system, we can consider a new state variable composed by a couple. In this couple, the first component is a real variable carrying the present of the

\footnotetext{
${ }^{1}$ Dipartimento di Scienze Economiche ed Aziendali, Facoltà di Economia, Libera Università internazionale degli studi sociali "Guido Carli", viale Romania 32, 00197 Roma, Italy. Email: sfederico@luiss.it.

${ }^{2}$ Center of Mathematics for Applications (CMA), University of Oslo, Box 1053 Blindern, NO- 0316 Oslo, Norway. Email: oksendal@math.uio.no.

${ }^{3}$ Norwegian School of Economics and Business Administration (NHH), Helleveien 30, NO-5045 Bergen, Norway.
} 
system, the second one is a functional variable carrying the past of the system. In this way, we can write the dynamics of this new infinite-dimensional state variable in a functional space, gaining markovianity and, on the other hand, paying the price of working in infinite dimension. For this kind of approach also in the context of control problems we refer to $[7,8,23]$ in the deterministic case and to $[1,3,6,10,11]$ in the stochastic Brownian case. To the best of our knowledge, despite the statement of a result in [19], this kind of approach was not developed for delay systems driven by a Lévy noise.

Nonetheless, in some cases still very interesting for the applications, it happens that systems with delay can be reduced to finite-dimensional systems, since the information we need from their dynamics can be represented by a finite-dimensional variable evolving in terms of itself. In such a context, the crucial point is to understand when this finite-dimensional reduction of the problem is possible and/or to find conditions ensuring that. There are some papers dealing with this subject in the stochastic Brownian case: we refer to $[5,12,13,16]$. The paper [4] represents an extension of [13] to the case when the equation is driven by a Lévy noise.

To some extent, our paper inserts in between these two approaches. What we do here is to rewrite the problem in infinite dimension and look for conditions yielding explicit solvability for it. It will turn out that, under these conditions, the problem can be reduced to a one-dimensional problem. In this sense the infinite-dimensional representation may be viewed as a tool to get in a fast and elegant way these conditions (we refer to Remark 4.3 for more comments on that). We think and hope that this paper may contribute to open a new way in this field: the infinite dimesional approach to delay problems can be used to get sufficient (and maybe necessary) conditions ensuring a finite-dimensional reduction of some classes of problems (along the line of what is done, by a direct finite-dimensional approach, in $[4,13])$.

\section{The optimal stopping problem}

Let $(\Omega, \mathcal{F}, P)$ be a probability space. Let $(B(t))_{t \geq 0},(\eta(t))_{t \geq 0}$ be two stochastic processes defined on $(\Omega, \mathcal{F}, P)$ such that

- $(B(t))_{t \geq 0}$ and $(\eta(t))_{t \geq 0}$ are independent;

- $(B(t))_{t \geq 0}$ is a standard Brownian motion with respect to its natural filtration;

- $(\eta(t))_{t \geq 0}$ is a pure jump Lévy process.

Define the Poisson random measure $N(t)$ of $\eta$ by

$$
N(t, A)=\sum_{s \in[0, t]} I_{A}\left(\eta(s)-\eta\left(s^{-}\right)\right), \quad A \subset \mathbb{R}
$$

and suppose that the Lévy measure $\nu$ of $\eta$ is such that

$$
\int_{\mathbb{R}} z^{2} \nu(d z)<+\infty
$$

This is equivalent to assume

$$
E\left[\eta(t)^{2}\right]<+\infty, \quad \forall t \geq 0 .
$$

Since $(\eta(t))_{t \geq 0}$ is a pure jump process we have $\nu(\{0\})=0$. Also define the measure-valued process $(\tilde{N}(t))_{t \geq 0}$ by

$$
\tilde{N}(t, d z)=N(t, d z)-\nu(d z),
$$


so that

$$
\tilde{N}(d t, d z)=N(d t, d z)-\nu(d z) d t .
$$

Then, for every $A \subset \mathbb{R}$ with $0 \notin \bar{A}, \tilde{N}(\cdot, A)$ is a square integrable martingale with respect to the natural filtration of $N$.

We denote by $\left(\mathcal{F}_{t}\right)_{t \geq 0}$ the filtration generated by $(B, \eta)$. Of course, due to the independence of $B$ and $\eta$, the processes $B(\cdot)$ and $\tilde{N}(\cdot ; A)$ are still respectively a Brownian motion and a square integrable martingale with respect to $\left(\mathcal{F}_{t}\right)_{t \geq 0}$.

Now let us define the Hilbert spaces

$$
L_{-T}^{2}:=L^{2}([-T, 0] ; \mathbb{R}), \quad H:=\mathbb{R} \times L_{-T}^{2} .
$$

and denote the inner product on $H$ by $\langle\cdot, \cdot\rangle$, i.e.

$$
\langle\cdot, \cdot\rangle=\langle\cdot, \cdot\rangle_{\mathbb{R}}+\langle\cdot, \cdot\rangle_{L_{-T}^{2}},
$$

and by $\|\cdot\|=\langle\cdot, \cdot\rangle^{1 / 2}$ the corresponding norm.

Also let us define the subspace of $H$

$$
\mathcal{C}:=\left\{y \in H \mid y_{1}(\cdot) \text { admits a càdlàg representative }\right\}
$$

When $y \in \mathcal{C}$ we will always consider the (pointwise well defined) càdlàg representative of $y_{1}(\cdot)$.

We are involved with the problem of the optimal stopping of the stochastic delay differential equation on the space $(\Omega, \mathcal{F}, P)$

$$
\left\{\begin{aligned}
d x(t)=\left[\begin{array}{l}
\left.\mu_{0} x(t)+\int_{-T}^{0} \mu_{1}(\xi) x(t+\xi) d \xi+\mu_{2} x(t-T)\right] d t+\left[\sigma_{0} x(t)+\int_{-T}^{0} \sigma_{1}(\xi) x(t+\xi) d \xi\right] d B(t) \\
\quad+\int_{\mathbb{R}}\left[\gamma_{0}(z) x\left(t^{-}\right)+\int_{-T}^{0} \gamma_{1}(z, \xi) x\left(t^{-}+\xi\right) d \xi\right] \tilde{N}(d t, d z),
\end{array}\right. \\
x(0)=y_{0} ; \quad x(s)=y_{1}(s), s \in[-T, 0) ;
\end{aligned}\right.
$$

where $y:=\left(y_{0}, y_{1}(\cdot)\right) \in \mathcal{C}$.

In the equation above $\mu_{2} \in \mathbb{R}, \mu:=\left(\mu_{0}, \mu_{1}(\cdot)\right) \in H, \sigma:=\left(\sigma_{0}, \sigma_{1}(\cdot)\right) \in H, \gamma(\cdot):=$ $\left(\gamma_{0}(\cdot), \gamma_{1}(\cdot)(\cdot)\right) \in L^{2}(\mathbb{R}, \nu ; H)$ are (functional) parameters.

A solution to (1) is a càdlàg adapted process $x(\cdot)$ satisfying the integral equation

$$
\begin{aligned}
x(t)=\eta_{0}+\int_{0}^{t}\left[\mu_{0} x(s)\right. & \left.+\int_{-T}^{0} \mu_{1}(\xi) x(s+\xi) d \xi+\mu_{2} x(s-T)\right] d s \\
& +\int_{0}^{t}\left[\sigma_{0} x(s)+\int_{-T}^{0} \sigma_{1}(\xi) x(s+\xi) d \xi\right] d B(s) \\
& +\int_{0}^{t} \int_{\mathbb{R}}\left[\gamma_{0}(z) x\left(s^{-}\right)+\int_{-T}^{0} \gamma_{1}(z, \xi) x\left(s^{-}+\xi\right) d \xi\right] \tilde{N}(d s, d z),
\end{aligned}
$$

where $x(\xi)=\eta_{1}(\xi)$ for $\xi \in[-T, 0)$. Straight from [20], Chapter V, Theorem 7 , we get the following.

Theorem 2.1. For every $y \in \mathcal{C}$, equation (1) admits a unique solution. 
Let us denote by $x(\cdot ; y)$ the unique solution to (1). The optimization problem consists in maximizing, over the set of the allowed stopping times $\tau \geq 0$, the functional

$$
J(y ; \tau):=E\left[e^{-\rho \tau} G\left(b_{0} x(\tau ; y)+\int_{-T}^{0} b_{1}(\xi) x(\tau+\xi ; y) d \xi\right)\right],
$$

where $\rho>0, b:=\left(b_{0}, b_{1}(\cdot)\right) \in H$ and $G$ is a real function. By sake of simplicity we restrict the set $\mathcal{T}$ to almost surely finite stopping times, i.e.

$$
\mathcal{T}=\left\{\tau \text { is }\left(\mathcal{F}_{t}\right)_{t \geq 0}-\text { stopping time, } \tau<+\infty \text { a.s. }\right\} .
$$

The value function associated to the problem is

$$
V(y)=\sup _{\tau \in \mathcal{T}} J(y ; \tau)
$$

\section{Reformulation in infinite dimension}

In this section we rewrite formally the problem in the space $H$ and show the equivalence with the originary one-dimensional delay problem.

Consider the unbounded operator $A$ defined on

$$
D(A)=\left\{y=\left(y_{0}, y_{1}(\cdot)\right) \in H \mid y_{1}(\cdot) \in W^{1,2}([-1,0] ; \mathbb{R}), y_{0}=y_{1}(0)\right\}
$$

by

$$
A y=\left(\mu_{0} y_{0}+\int_{-T}^{0} \mu_{1}(\xi) y_{1}(\xi) d \xi+\mu_{2} y_{1}(-T), y_{1}^{\prime}(\cdot)\right)
$$

It is well-known that $A$ is the generator of a strongly continuous semigroup $(S(t))_{t \geq 0}$ on $H$. More precisely $S(t)$ acts in this way on $H$ :

$$
S(t) y=\left(u(t ; y),\left.u(t+\xi ; y)\right|_{\xi \in[-T, 0]}\right),
$$

where $u(\cdot ; y)$ is the solution of

$$
\left\{\begin{array}{l}
u^{\prime}(t)=\left[\mu_{0} u(t)+\int_{-T}^{0} \mu_{1}(\xi) u(t+\xi) d \xi+\mu_{2} u(t-T)\right] \\
u(0)=y_{0}, \quad u(s)=y_{1}(s), s \in[-T, 0) .
\end{array}\right.
$$

We can define the following linear stochastic evolution equation in the space $H$ :

$$
\left\{\begin{array}{l}
d X(t)=A X(t) d t+\langle\sigma, X(t)\rangle \cdot \hat{n} d B(t)+\int_{\mathbb{R}}\left\langle\gamma(z), X\left(t^{-}\right)\right\rangle \cdot \hat{n} \tilde{N}(d t, d z), \\
X(0)=y
\end{array}\right.
$$

where $\hat{n}=(1,0) \in H$ and $X\left(t^{-}\right):=\lim _{s \uparrow t} X(s)$, with the limit taken in the space $H$.

At least formally (6) should represent (1) in the space $H$, in the sense that

$$
X(t)=\left(X_{0}(t), X_{1}(t)\right)=\left(x(t),\left.x(t+\xi)\right|_{\xi \in[-T, 0]}\right), \quad \forall t \geq 0 .
$$

To prove that we need to do some work to study the infinite-dimensional state equation. 


\subsection{The state equation in the space $H$ : existence and uniqueness of mild solutions}

In this subsection we study the state equation (6) in the space $H$. First of all we sketch some results about the integration of $H$-valued process. Notice that in (6) the differentials are just with respect to one-dimensional noises.

Let

$$
\begin{aligned}
& \mathbb{D}_{H}^{2}=\{Y:[0,+\infty) \times \Omega \rightarrow H \mid \\
& \qquad(\cdot, \omega) \text { is }\left(\mathcal{F}_{t}\right)_{t \geq 0} \text {-adapted and càdlàg for a.e. } \omega \in \Omega, \\
& \left.\qquad E\left[\int_{0}^{t}\|Y(s)\|^{2} d s\right]<+\infty \forall t \geq 0\right\} .
\end{aligned}
$$

As in the finite-dimensional case we can define the stochastic integral process with repect to $d B(s)$ for a process $Y \in \mathbb{D}_{H}^{2}$,

$$
I_{B}(Y)(t)=\int_{0}^{t} Y(s) d B(s)
$$

Now let

$$
\begin{aligned}
& \mathbb{L}_{H}^{2}(\mathbb{R})=\{Z:[0,+\infty) \times \mathbb{R} \times \Omega \rightarrow H \mid \\
& \qquad(\cdot, z, \omega) \text { is }\left(\mathcal{F}_{t}\right)_{t \geq 0} \text {-adapted and càglàd for a.e. } z \in \mathbb{R}, \omega \in \Omega, \\
& \left.\qquad E\left[\int_{0}^{t} \int_{\mathbb{R}}\|Z(s)\|^{2} \nu(d z) d s\right]<+\infty \forall t \geq 0\right\} .
\end{aligned}
$$

As in the finite-dimensional case we can define the stochastic integral process with repect to $\tilde{N}(d s, d z)$ for a process $Z \in \mathbb{L}_{H}^{2}(\mathbb{R})$,

$$
I_{\tilde{N}}(Z)(t)=\int_{0}^{t} \int_{\mathbb{R}} Z(s, z) \tilde{N}(d s, d z) .
$$

As in the finite-dimensional case, it turns out that the processes defined in (8) and (10) are square integrable $H$-valued martingales, that there exists a continuous version for $I_{B}(Y)$ and a càdlàg version for $I_{\tilde{N}}(Z)$, and that the Ito-Lévy isometry holds, i.e., for every $t \geq 0$,

$$
E\left[\left(I_{B}(Y)(t)\right)^{2}\right]=E\left[\int_{0}^{t}\|Y(s)\|^{2} d s\right], \quad E\left[\left(I_{\tilde{N}}(Z)(t)\right)^{2}\right]=E\left[\int_{0}^{t} \int_{\mathbb{R}}\|Z(s, z)\|^{2} \nu(d z) d s\right] .
$$

Let us give the definition of a mild solution to (6).

Definition 3.1. Let $y \in H$. A process $X \in \mathbb{D}_{H}^{2}$ is called a mild solution to (6) if

$$
\begin{aligned}
X(t)=S(t) y+\int_{0}^{t}\langle\sigma, X(t-\tau)\rangle & S(t-\tau) \hat{n} d B(\tau) \\
& +\int_{0}^{t} \int_{\mathbb{R}}\left\langle\gamma(z), X\left((t-\tau)^{-}\right)\right\rangle S(t-\tau) \hat{n} \tilde{N}(d \tau, d z), \quad \forall t \geq 0 .
\end{aligned}
$$


Theorem 3.2. Equation (6) admits a unique mild solution $X(\cdot ; y) \in \mathbb{D}_{H}^{2}$.

Proof. Due to the Ito-Lévy isometry and to the fact that $S(\cdot)$ is a strongly continuous semigroup on $H$, the proof follows exactly the line of the finite-dimensional case.

Remark 3.3. Note that in Definition 3.1 we have used the definition

$$
X\left(t^{-}\right):=\lim _{s \uparrow t} X(s)=\left(\lim _{s \uparrow t} X_{0}(s), \lim _{s \uparrow t} X_{1}(s)\right)
$$

where the limit in the first component of the right hand side is taken in $\mathbb{R}$ and the one in the second component is taken in $L_{-T}^{2}$. This should not a priori be assumed to be the same as

$$
\left(X_{0}\left(t^{-}\right), X_{1}(t)\left(\xi^{-}\right)\right)=\left(\lim _{s \uparrow t} X_{0}(t), \lim _{\eta \uparrow \xi} X_{1}(t)(\eta)\right), \quad \xi \in[-T, 0],
$$

where the limit in the second component in the right hand side is taken in $\mathbb{R}$, for $\xi \in[-T, 0]$ fixed. In particular,

$$
X_{1}(t)\left(\xi^{-}\right)=\lim _{\eta \uparrow \xi} X_{1}(t)(\eta)
$$

is not well-defined, since, for fixed $t$, the function

$$
[-T, 0] \ni \xi \mapsto X_{1}(t)(\xi)
$$

is not well-defined pointwise (it is only an element of $L_{-T}^{2}$, i.e. we only know its equivalence class). Nevertheless (12) can be given a rigorous interpretation, under which (12) coincides with (11) (see Remark 3.8).

\subsection{The state equation in the space $H$ : weak solutions}

In this subsection we investigate a different concept of solution for equation (6), i.e. the concept of weak solution. It will turn out to be very useful in the following. First we introduce the operator $A^{*}$, i.e. the adjoint of the operator $A$. It is defined on

$$
D\left(A^{*}\right)=\left\{y=\left(y_{0}, y_{1}(\cdot)\right) \in H \mid y_{1} \in W^{1,2}([-T, 0] ; \mathbb{R}), y_{1}(-T)=\mu_{2} y_{0}\right\},
$$

by

$$
A^{*} y=\left(\mu_{0} y_{0}+y_{1}(0), \mu_{1}(\cdot) y_{0}-y_{1}^{\prime}(\cdot)\right) .
$$

Definition 3.4. Let $y \in H$. A process $X \in \mathbb{D}_{H}^{2}$ is called a weak solution to (6) if

$$
\begin{aligned}
\langle X(t), h\rangle=\langle y, h\rangle+\int_{0}^{t}\langle X(s), & \left.A^{*} h\right\rangle d s+\int_{0}^{t}\langle\sigma, X(s)\rangle\langle\hat{n}, h\rangle d B(s) \\
& +\int_{0}^{t} \int_{\mathbb{R}}\left\langle\gamma(z), X\left(s^{-}\right)\right\rangle\langle\hat{n}, h\rangle \tilde{N}(d s, d z), \quad \forall t \geq 0, \forall h \in D\left(A^{*}\right) .
\end{aligned}
$$

In this case the two concepts of solution we have given coincide.

Proposition 3.5. Let $y \in H$. A process $X \in \mathbb{D}_{H}^{2}$ is a weak solution of (6) if and only if it is a mild solution of $(6)$.

Proof. See [19], Theorem 9.15. 


\subsection{Equivalence between the original delay problem and the infinite dimen- sional one}

To give sense to our approach we must show that the infinite-dimensional problem defined in the subsection above represents the original delay problem. We start with a lemma.

Lemma 3.6. Let $y \in \mathcal{C}$ and let $x(\cdot):=x(\cdot ; y)$ be the unique solution to (1). Moreover let $u^{0}(\cdot):=u(\cdot ;(1,0))$ be the unique solution to $(5)$ with initial datum $(1,0)$ and let $u(\cdot ; y)$ be the unique solution to (5) with initial datum $y$. Then $x(\cdot)$ satisfies the variation of constants formula

$$
\begin{aligned}
x(t) & =u(t ; y)+\int_{0}^{t}\left[\sigma_{0} x(t-\tau)+\int_{-T}^{0} \sigma_{1}(\xi) x(t-\tau+\xi) d \xi\right] u^{0}(t-\tau) d B(t) \\
& +\int_{0}^{t} \int_{\mathbb{R}}\left[\gamma_{0}(z) x\left((t-\tau)^{-}\right)+\int_{-T}^{0} \gamma_{1}(z, \xi) x\left((t-\tau+\xi)^{-}\right) d \xi\right] u^{0}(t-\tau) \tilde{N}(d t, d z), \quad t \geq 0 .
\end{aligned}
$$

Proof. See Theorem 1.1 in [22].

Now we can state our equivalence result.

Theorem 3.7. Let $y \in \mathcal{C}$ and let $x(\cdot ; y), X(\cdot ; y)$ be respectively the solution to (1) and the mild solution to $(6)$. Then $X(\cdot ; y)$ represents $x(\cdot ; y)$, in the sense that

$$
X(t ; y)=\left(x(t ; y),\left.x(t+\xi ; y)\right|_{\xi \in[-T, 0]}\right), \quad \forall t \geq 0 .
$$

Proof. By uniqueness we should only check that $\left(x(t ; y),\left.x(t+\xi ; y)\right|_{\xi \in[-T, 0]}\right)$ is a mild solution of (6). Therefore, the proof is a straight application of Lemma 3.6 and of (4)-(5).

Remark 3.8. The result above in particular shows that $X(t ; y) \in \mathcal{C}$ for every $t \geq 0$ (in the sense that almost surely there exists a (unique) representative of $X(t ; y)$ in the space $H$ for every $t \geq 0)$. Given such representation for $X(\cdot ; y)$, we have

$$
X\left(t^{-}\right)=\lim _{s \uparrow t}(x(s), x(s+\cdot))=\left(x\left(t^{-}\right),\left.x\left(t+\xi^{-}\right)\right|_{\xi \in[-T, 0]}\right)=\left(X_{0}\left(t^{-}\right),\left.X_{1}(t)\left(\xi^{-}\right)\right|_{\xi \in[-T, 0]}\right),
$$

where the limit is taken in the space $H$. The equality in the middle is true by continuity of translations in $L^{2}(\mathbb{R})$.

Thanks to Theorem 3.7, the functional (2) can be rewritten in the infinite-dimensional setting as

$$
J(y ; \tau)=E\left[e^{-\rho \tau} G(\langle b, X(\tau ; y)\rangle)\right]
$$

\subsection{The infinitesimal operator of $X(\cdot ; y)$}

The infinitesimal operator of the mild solution $X(\cdot ; y)$ of $(6)$ is formally defined as

$$
\begin{aligned}
{[\mathcal{L} \varphi](y) } & :=\left\langle A y, \varphi_{y}(y)\right\rangle+\frac{1}{2}\langle\sigma, y\rangle \varphi_{y_{0} y_{0}}(y) \\
& +\int_{\mathbb{R}}\left[\varphi(y+\langle\gamma(z), y\rangle \hat{n})-\varphi(y)-\varphi_{y_{0}}(y)\langle\gamma(z), y\rangle\right] \nu(d z), \quad y \in D(A), \varphi \in C^{2}(H ; \mathbb{R}) .
\end{aligned}
$$


In order to remove the requirement $y \in D(A)$, we require more regularity for the functions belonging to the domain of $\mathcal{L}$, i.e. $\varphi_{y} \in C\left(H, D\left(A^{*}\right)\right)$. Then we can rewrite $\mathcal{L}$ as

$$
\begin{aligned}
{[\mathcal{L} \varphi](y):=} & \left\langle y, A^{*} \varphi_{y}(y)\right\rangle+\frac{1}{2}\langle\sigma, y\rangle \varphi_{y_{0} y_{0}}(y) \\
& +\int_{\mathbb{R}}\left[\varphi(y+\langle\gamma(z), y\rangle \hat{n})-\varphi(y)-\varphi_{y_{0}}(y)\langle\gamma(z), y\rangle\right] \nu(d z), \quad y \in H, \varphi \in C^{2}(H ; \mathbb{R}) .
\end{aligned}
$$

\section{The free boundary problem in the space $H$}

We can write formally the free boundary problem associated with our optimization problem in the space $H$. Inspired by the finite-dimensional case, we see that, if we want to solve the optimization problem, we should be able to find $v \in C^{1}(H)$ and an open set $\mathcal{O} \subset H$ such that

$$
\begin{cases}\text { (i) } v \in C^{2}(H \backslash \partial \mathcal{O}), & v_{x x} \text { bounded on } H \backslash \partial \mathcal{O}, \\ \text { (ii) } \mathcal{L} v \leq \rho v, & \text { on } H \backslash \partial \mathcal{O}, \\ \text { (iii) } \mathcal{L} v=\rho v, & \text { on } \mathcal{O}, \\ \text { (iv) } v=G(\langle b, \cdot\rangle), & \text { on } \mathcal{O}^{c}, \\ \text { (v) } v>G, & \text { on } \mathcal{O},\end{cases}
$$

where $\mathcal{O}$ is an open set of $H$. We want to look for conditions on the parameters of the model yielding the possibility of finding explicit solutions to (16).

Due to the structure of condition (iv) in (16), we guess that, at least under some assumptions that we are going to derive, the problem (16) admits a solution in the form

$$
\left\{\begin{array}{l}
\mathcal{O}=\{y \in H \mid\langle b, y\rangle \in \mathcal{A}\}, \quad \mathcal{A} \subset \mathbb{R} \text { open, } \\
v=f(\langle b, \cdot\rangle) \text { on } \mathcal{O}
\end{array}\right.
$$

where $f \in C^{2}(\mathcal{A} ; \mathbb{R})$.

Remark 4.1. We can guess that, for suitable choices of $G$, the set $\mathcal{A}$ in (17) takes the form

$$
\mathcal{A}=\left(c_{0},+\infty\right),
$$

where $c_{0} \in[0,+\infty)$.

\subsection{The equation $\mathcal{L} v=\rho v$}

Here we derive conditions on the parameters for which the equation $\mathcal{L} v=\rho v$ admits explicit solutions in the form $f(\langle b, \cdot\rangle), f \in C^{2}(\mathbb{R} ; \mathbb{R})$. Inspired by the one-dimensional case and due to the structure of $\mathcal{L}$, we look for solutions in the form above with $f(z)=z^{a}, a \in \mathbb{R}$. So, consider

$$
w(y)=\langle b, y\rangle^{a} \text {. }
$$

Putting this expression inside equation (16)-(iii), we get

$$
\begin{aligned}
a\langle b, y\rangle^{a-1}\left\langle y, A^{*} b\right\rangle & +\frac{1}{2}\langle\sigma, y\rangle^{2} a(a-1)\langle b, y\rangle^{a-2} \sigma_{0}^{2} \\
& +\int_{\mathbb{R}}\left[\langle y+z\langle\gamma, y\rangle \hat{n}, b\rangle^{a}-\langle y, b\rangle^{a}-a \gamma_{0} z\langle b, y\rangle^{a-1}\langle\gamma, y\rangle\right] \nu(d z)=\rho\langle b, y\rangle^{a} .
\end{aligned}
$$


Therefore, we see that $w$ can be a solution of (16)-(iii) imposing

$$
\left\{\begin{array}{l}
\text { (i) } \sigma=\alpha b, \text { for some } \alpha \in \mathbb{R}, \\
\text { (ii) } \gamma(z)=\beta(z) b, \text { for some } \beta(\cdot) \in L^{2}(\mathbb{R}, \nu), \\
\text { (iii) } b \in D\left(A^{*}\right), \\
\text { (iv) } A^{*} b=\lambda b, \text { for some } \lambda \in \mathbb{R} .
\end{array}\right.
$$

Let us suppose that (20) are satisfied and let us check when $w$ is actually a solution of (16)-(iii). Due to (20), equation (19) above can be rewritten as

$$
a \lambda\langle b, y\rangle^{a}+\frac{1}{2} a(a-1) \alpha^{2} b_{0}^{2}\langle b, y\rangle^{a}+\left(\int_{\mathbb{R}}\left(\left(1+b_{0} \beta(z)\right)^{a}-1-a \gamma_{0} \beta(z)\right) \nu(d z)\right)\langle b, y\rangle^{a}=\rho\langle b, y\rangle^{a} .
$$

Set

$$
h(a, \lambda)=-\rho+a \lambda+\frac{1}{2} a(a-1) \alpha^{2} b_{0}^{2}+\int_{\mathbb{R}}\left(\left(1+b_{0} \beta(z)\right)^{a}-1-a b_{0} \beta(z)\right) \nu(d z) .
$$

Suppose that there exists a solution $a^{*}(\lambda)$ to

$$
h(\cdot, \lambda)=0
$$

This implies that, putting the value $a^{*}(\lambda)$ in the expression of $w$ given in (18), it solves (16)-(iii).

Now let us see when conditions (20)-(iii) and (20)-(iv) are satisfied. From (13) we see that (20)-(iii) yields

$$
\left\{\begin{array}{l}
b_{1} \in W^{1,2}([-T, 0] ; \mathbb{R}), \\
b_{1}(-T)=a_{2} b_{0} .
\end{array}\right.
$$

From (14) we see that (20)-(iv) yields

$$
\left\{\begin{array}{l}
\mu_{0} b_{0}+b_{1}(0)=\lambda b_{0} \\
b_{1}^{\prime}(\cdot)=\mu_{1}(\cdot) b_{0}-\lambda b_{1}(\cdot)
\end{array}\right.
$$

Therefore, from the second condition of (23) we get

$$
b_{1}(s)=b_{1}(0) e^{-\lambda s}-b_{0} \int_{s}^{0} e^{-\lambda(s-\xi)} \mu_{1}(\xi) d \xi, \quad s \in[-T, 0] .
$$

Taking into account the first condition of (23) and (24) we get

$$
b_{1}(s)=b_{0}\left[\left(\lambda-\mu_{0}\right) e^{-\lambda s}-\int_{s}^{0} e^{-\lambda(s-\xi)} \mu_{1}(\xi) d \xi\right], \quad s \in[-T, 0] .
$$

Finally from the second condition of $(22)$ we get

$$
\mu_{2}=\left(\lambda-\mu_{0}\right) e^{\lambda T}-\int_{-T}^{0} e^{\lambda(T+\xi)} \mu_{1}(\xi) d \xi .
$$

Therefore, starting from data $\mu=\left(\mu_{0}, \mu_{1}(\cdot)\right) \in H, \mu_{2} \in \mathbb{R}$, whenever (26) admits a solution $\lambda^{*} \in \mathbb{R}$, we see that conditions (20)-(i,ii) and (25) provide the existence of a solution for equation (16)-(iii) given by

$$
w(y)=\langle b, y\rangle^{a^{*}}
$$


where $a^{*}:=a^{*}\left(\lambda^{*}\right)$ is a solution of $(21)$ with $\lambda=\lambda^{*}$.

Therefore, whenever

$$
\left\{\begin{array}{l}
(26) \text { admits a solution } \lambda^{*}, \\
b=\left(b_{0}, b_{1}(\cdot)\right) \text { satisfies }(25) \text { with } \lambda=\lambda^{*}, \\
\sigma=\alpha b, \quad \alpha \in \mathbb{R}, \\
\gamma(z)=\beta(z) b, \quad \beta(\cdot) \in L^{2}(\mathbb{R}, \nu),
\end{array}\right.
$$

the function (27) solves (16)-(iii).

Hence, given (28), a general solution to (16)-(iii) is

$$
\varphi(y)=C_{1}\langle b, y\rangle^{a_{1}^{*}}+\ldots+C_{n}\langle b, y\rangle^{a_{n}^{*}},
$$

where the $a_{i}^{*}$ 's, $i=1, \ldots, n$, are all the possible solutions of (21) (supposing they are finite) corresponding to $\lambda^{*}$ solution of (26).

Remark 4.2. When $\mu_{1}(\cdot) \equiv 0$, a straightforward qualitative study of the function

$$
\lambda \mapsto\left(\lambda-\mu_{0}\right) e^{\lambda T}
$$

shows that (26) admits a solution whenever

$$
\mu_{2} \in\left[-\frac{1}{T} e^{-\frac{1}{T}+\mu_{0}},+\infty\right)
$$

Moreover, if $\mu_{2} \in[0,+\infty)$, this solution is unique. Uniqueness of such a solution means that, starting from $\mu$, we can construct just one $b$ such that the problem has a solution in the form described above.

\subsection{The state equation and the natural boundary of $X(\cdot ; y)$}

In this subsection we study the properties of the state equation when conditions (20) are satisfied. In this case the state equation in infinite dimension reads as

$$
\left\{\begin{array}{l}
d X(t)=A X(t) d t+\alpha\langle b, X(t)\rangle \cdot \hat{n} d B(t)+\int_{\mathbb{R}} \beta(z)\langle b, X(t)\rangle \tilde{N}(d t, d z), \\
X(0)=y
\end{array}\right.
$$

We still denote the unique mild solution of $(31)$ by $X(\cdot ; y)$. We are interested to see the behaviour of the infinite-dimensional diffusion $X(\cdot ; y)$ with respect to the sets

$$
\mathcal{G}_{0}=\{y \in H \mid\langle b, y\rangle=0\}, \quad \mathcal{G}_{+}=\{y \in H \mid\langle b, y\rangle>0\}, \quad \mathcal{G}_{-}=\{y \in H \mid\langle b, y\rangle<0\} .
$$

Let $y \in H$ and set $X(\cdot):=X(\cdot ; y)$. Since $X(\cdot)$ is a mild solution of (6), due to Proposition 3.5, it is also a weak solution of (31). Therefore, taking into account (20)-(iii) and (20)-(iv), taking $h=b$ in the definition of weak solution we get

$$
\begin{aligned}
\langle X(t), b\rangle=\langle y, b\rangle+\lambda \int_{0}^{t}\langle X(s), b\rangle d s+\alpha b_{0} \int_{0}^{t}\langle & X(s), b\rangle d B(s) \\
& +b_{0} \int_{0}^{t} \int_{\mathbb{R}} \beta(z)\langle X(s), b\rangle \tilde{N}(d s, d z), \quad \forall t \geq 0 .
\end{aligned}
$$


Then we see that the one-dimensional process $Z(\cdot ; y):=\langle X(\cdot), b\rangle$ is a geometric Lévy process starting from $\langle y, b\rangle$. Therefore, supposing

$$
b_{0} \beta(z)>-1, \quad \nu-\text { a.s. }
$$

we have

$$
\left\{\begin{array}{l}
\left(\text { i) } \quad y \in \mathcal{G}_{0} \Longrightarrow X(t ; y) \in \mathcal{G}_{0}, \forall t \geq 0,\right. \\
\text { (ii) } y \in \mathcal{G}_{+} \Longrightarrow X(t ; y) \in \mathcal{G}_{+}, \forall t \geq 0, \\
\text { (iii) } y \in \mathcal{G}_{-} \Longrightarrow X(t ; y) \in \mathcal{G}_{-}, \forall t \geq 0 .
\end{array}\right.
$$

Due to (33), according to Feller's boundary classification (for diffusions), we could say that $\mathcal{G}_{0}$ is a natural boundary for the process $X(\cdot ; y)$.

Remark 4.3. We see that, when (20) are satisfied, we can describe the evolution of the onedimensional variable $Z(\cdot ; y):=\langle X(\cdot ; y), b\rangle$ in terms of a one-dimensional stochastic differential equation. If the objective functional can be written in terms of this variable $Z$ as well (as in our case), then the problem reduces to a one-dimensional problem. The question is: how to derive the conditions (20) which ensure this reduction? Is the infinite-dimensional representation really necessary?

It is not clear how to derive such conditions working just with the one-dimensional delay equation (1), so that the infinite-dimensional representation seems to be, at least, very convenient. However, we should also say that we could derive these conditions working on the state equation in infinite dimension rather than on the differential problem (16)-(iii). Indeed, due to Proposition 3.5 , the mild solution $X(\cdot ; y)$ to $(6)$ is also a weak solution so that

$$
\begin{aligned}
\langle X(t), h\rangle=\langle y, h\rangle+\int_{0}^{t}\langle X(s), & \left.A^{*} h\right\rangle d s+\int_{0}^{t}\langle\sigma, X(s)\rangle\langle\hat{n}, h\rangle d B(s) \\
& +\int_{0}^{t} \int_{\mathbb{R}}\left\langle\gamma(z), X\left(s^{-}\right)\right\rangle\langle\hat{n}, h\rangle \tilde{N}(d s, d z), \quad \forall t \geq 0, \forall h \in D\left(A^{*}\right)
\end{aligned}
$$

and we see that to impose that $\langle X(\cdot), h\rangle$ evolves in terms of itself corresponds exactly to require (20). We have chosen to work with the differential problem (16)-(iii) because the system (16) should work as (formal) differential problem associated to the optimal stopping problem also when (20) are not verified. This leaves open the investigation of other less natural cases by (16).

\subsection{The free boundary conditions}

To solve the free boundary problem (16) we must impose the conditions (iii,iv) of (16) to the general solution (29) found in Subsection 4.1.

So, conditions (iv,v) in (16) and condition (17) yield

$$
\begin{cases}\text { (i) } G(z)=C_{1} z^{a_{1}^{*}}+\ldots+C_{n} z^{a_{n}^{*}}, & \text { on } \partial \mathcal{A}, \\ \text { (ii) } G(z)<C_{1} z^{a_{1}^{*}}+\ldots+C_{n} z^{a_{n}^{*}}, & \text { on } \mathcal{A} .\end{cases}
$$

Moreover the requirement $v \in C^{1}(H)$ yields the smooth fit principle

$$
\left\{\begin{array}{l}
(i) \exists G^{\prime} \quad \text { on } \partial \mathcal{A}, \\
(\text { ii }) G^{\prime}(z)=C_{1} a_{1}^{*} z^{a_{1}^{*}-1}+\ldots+C_{n} a_{n}^{*} z^{a_{n}^{*}-1}, \text { on } \partial \mathcal{A} .
\end{array}\right.
$$




\section{Verification Theorem}

Assume conditions (28); moreover, assume that (21) has solutions $a_{1}^{*}, \ldots, a_{n}^{*}$ and that (34) and (35) have a solution $\left(\mathcal{A}^{*} ; C_{1}^{*}, \ldots, C_{n}^{*}\right)$. Let

$$
\mathcal{O}^{*}=\left\{y \in H \mid\langle b, y\rangle \in \mathcal{A}^{*}\right\}
$$

Under these requirements, let $v$ be the solution to the free boundary problem (16) constructed in the previous section. It is characterized by $\mathcal{A}^{*}, a_{1}^{*}, \ldots, a_{n}^{*}, C_{1}^{*}, \ldots, C_{n}^{*}$ as

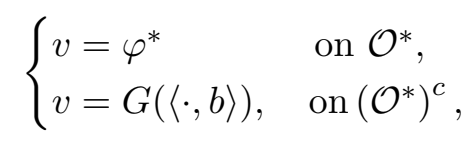

where $\varphi^{*}$ is the function defined in $(29)$ with $C_{1}=C_{1}^{*}, \ldots, C_{n}=C_{n}^{*}$. We want to show that $v$ is really the value function and that $\mathcal{O}^{*}$ is the continuation region for the problem.

Define the one-dimensional process $Z(\cdot ; y)=\langle X(\cdot ; y), b\rangle$. From Subsection 4.2 we know that $Z(\cdot ; y)$ is a geometric Lévy process starting at $\langle b, y\rangle$. We can rewrite the functional (2) as

$$
J(y ; \tau)=E\left[e^{-\rho \tau} G(Z(\tau ; y))\right] .
$$

Also we see that

$$
f(z)=C_{1}^{*} z^{a_{1}^{*}}+\ldots+C_{n}^{*} z^{a_{n}^{*}}
$$

solves

$$
\begin{cases}\frac{1}{2} \alpha^{2} b_{0}^{2} z^{2} f^{\prime \prime}(z)+\lambda^{*} z f^{\prime}(z)+\int_{\mathbb{R}}\left(\left(f(z+\beta(z))-f(z)-\beta(z) f^{\prime}(z)\right) \nu(d z)=\rho f(z), \quad z \in \mathcal{A}^{*},\right. \\ f=G, & \text { on } \partial \mathcal{A}^{*} \\ f^{\prime}=G^{\prime}, & \text { on } \partial \mathcal{A}^{*}, \\ f>G, & \text { on } \mathcal{A}^{*} .\end{cases}
$$

Therefore, arguing with $Z$ and $f$ and with the expression of $J$ given in (38), from the onedimensional case (see [16], Chapter 3) we get the following.

Theorem 5.1. Let conditions (28) hold. Moreover, assume that (21) has solutions $a_{1}^{*}, \ldots, a_{n}^{*}$ and that (34) and (35) have a solution $\left(\mathcal{A}^{*} ; C_{1}^{*}, \ldots, C_{n}^{*}\right)$. Let $v, \mathcal{O}^{*}$ be defined as in (37) and (36) respectively. Suppose that $v$ satisfies also (16)-(ii).

Then $v=V$ and $\mathcal{O}^{*}$ is the continuation region for the optimal stopping problem.

Remark 5.2. As a consequence of the Verification Theorem, we see that there exists at most one solution $\left(\mathcal{A}^{*} ; C_{1}^{*}, \ldots, C_{n}^{*}\right)$ to $(34)$-(35) such that the corresponding $v$ satisfies (16)-(ii).

\section{Applications: the American put option}

In this section we give an example of applications of our argument to the American put option problem. Suppose to have a stock dynamics driven by the following stochastic differential equation: 


$$
\left\{\begin{aligned}
& d x(t)=\mu_{0} {[x(t)-x(t-T)] d t+\alpha\left[x(t)-\mu_{0} \int_{-T}^{0} x(t+\xi) d \xi\right] d B(t) } \\
&+\beta \int_{\mathbb{R}}\left[x\left(t^{-}\right)-\mu_{0} \int_{-T}^{0} x\left(t^{-}+\xi\right) d \xi\right] z \tilde{N}(d t, d z), \\
& x(0)=y_{0} ; \quad x(s)=y_{1}(s), s \in[-T, 0) ;
\end{aligned}\right.
$$

where $\mu_{0}, \alpha, \beta \in \mathbb{R}$ and $y:=\left(y_{0}, y_{1}(\cdot)\right) \in \mathcal{C}$ is such that $y_{0}-\mu_{0} \int_{-T}^{0} y_{1}(\xi) d \xi>0$. Moreover suppose that

$$
\nu \equiv 0 \text { on }(-\infty, 0]
$$

i.e. only positive jumps can occur.

Suppose that the optimal stopping problem consists in optimizing over $\tau \in \mathcal{T}$ the functional

$$
E\left[\left(K-\left(x(\tau)-\mu_{0} \int_{-T}^{0} x(\tau+\xi) d \xi\right)\right)^{+}\right], \quad K>0
$$

Notice that, from Subsection 4.2, we see that $x(t)-\mu_{0} \int_{-T}^{0} x(t+\xi) d \xi>0$ for all $t \geq 0$, so that the functional above is bounded from above by $K$. Of course it is also bounded from below by 0 , so that the problem is well-defined.

With this settings, we are taking $\mu_{1}(\cdot) \equiv 0, \mu_{2}=-\mu_{0}, b_{0}=1, b_{1}(\cdot) \equiv-\mu_{0}, \lambda^{*}=0$ in $(28)$. In this case (21) reads as

$$
h^{0}(a):=-\rho+\frac{1}{2} a(a-1) \alpha^{2}+\int_{\mathbb{R}}\left((1+\beta z)^{a}-1-a \beta z\right) \nu(d z)=0 .
$$

We see that

$$
h^{0}(0)=-\rho<0, \quad \lim _{a \rightarrow-\infty} h^{0}(a)=+\infty,
$$

Therefore $h^{0}(a)=0$ admits a solution $a^{*} \in(-\infty, 0)$.

Let us suppose that this solution is unique (actually it could be proved a posteriori, by Verification, that it is really unique). Due to the structure of the problem we can guess that the region $\mathcal{A}$, candidate to be the solution, has the structure

$$
\mathcal{A}=\left(z_{0},+\infty\right), \quad z_{0} \in(0, K) .
$$

Imposing (34)-(35), we get the system

$$
\left\{\begin{array}{l}
C z_{0}^{a^{*}}=K-z_{0} \\
C a^{*} z_{0}^{a^{*}-1}=-1
\end{array}\right.
$$

This system admits a unique solution $\left(C^{*}, z_{0}^{*}\right)$ with $C^{*}>0, z_{0}^{*} \in(0, K)$.

Let $\mathcal{A}^{*}=\left(z_{0}^{*},+\infty\right), \mathcal{O}^{*}=\left\{y \in H \mid\langle b, y\rangle \in \mathcal{A}^{*}\right\}$. The function

$$
v(y)= \begin{cases}K-\langle b, y\rangle, & \text { if }\langle b, y\rangle \leq z_{0}^{*}, \\ C^{*}\langle b, y\rangle^{*}, & \text { if }\langle b, y\rangle>z_{0}^{*},\end{cases}
$$

satisfies the assumptions of Theorem 5.1 (see the analogous in [16], Chapter 3), therefore $v=V$ and $\mathcal{O}^{*}$ is the continuation region for the problem. 
Remark 6.1. If we relax assumption (41) only requring

$$
\nu \equiv 0 \text { on }(-\infty,-1]
$$

the problem can be solved as well. In this case we have to invoke Remark 4.3 and another result coming from the one-dimensional theory, i.e. the result on the perpetual American put option contained in [14]. Indeed, due to Remark 4.3, the problem of maximizing (42) under the state equation (40) can be rewritten as

$$
\left\{\begin{array}{l}
\text { Maximize } E\left[e^{-\rho \tau}(K-Z(\tau))^{+}\right] \\
\text {subject to }\left\{\begin{array}{l}
d Z(t)=\alpha Z(t) d B(t)+\beta \int_{\mathbb{R}} z Z(t) \tilde{N}(d t, d z), \\
Z(0)=y_{0}-\mu_{0} \int_{-T}^{0} y_{1}(\xi) d \xi>0
\end{array}\right.
\end{array}\right.
$$

and we can apply the results of [14].

Acknowledgements. This paper was written during the visit of Salvatore Federico at Centre of Mathematics for Application - University of Oslo (May-June, 2009). The visit was supported by a grant of European Science Fundation - AMaMeF. The author thanks both the institutions.

\section{References}

[1] Chojnowska-Michalik A., Representation theorem for general stochastic delay equations, Bull. Acad. Polon. Sci. Ser. Sci. Math. Astronom. Phys., 26, 7, pp. 635-642, 1978.

[2] Da Prato G., Zabczyk J., Stochastic Equations in Infinite Dimensions, Encyclopedia of Mathematics and its Applications, Cambridge University Press, Cambridge (UK), 1992.

[3] Da Prato G., Zabczyk J., Ergodicity for Infinite Dimensional Systems, London mathematical society lecture note series, Vol. 229, Cambridge University Press, 1996.

[4] David D., Optimal control of stochastic delayed systems with jumps, preprint, 2008.

[5] Elsanousi I., Øksendal B., Sulem A., Some solvable stochastic control problems with delay, Stochastics and Stochastics Reports, 71, pp 69-89, 2000.

[6] Federico S., A stochastic control problem with delay arising in a pension fund model, submitted to Finance and Stochastics, 2008.

[7] Federico S., Goldys B., Gozzi F., HJB Equations for the Optimal Control of Differential Equations with Delays and State Constraints, I: Regularity and Applications, submitted to SIAM Journal on Control and Optimization, ArXiv preprint, 2009.

[8] Federico S., Goldys B., Gozzi F., HJB Equations for the Optimal Control of Differential Equations with Delays and State Constraints, II: Optimal Feedbacks and Approximations, ArXiv preprint, 2009.

[9] Gapeev P., Reiss M., Optimal stopping problem in a diffusion-type model with delay, Stochastics and Probability Letters, 76, 6, pp. 601-608, 2006.

[10] Gozzi F., Marinelli C., Stochastic optimal control of delay equations arising in advertising models, Da Prato (ed.) et al., Stochastic partial differential equations and applications VII - Papers of the 7th meeting, Levico Terme, Italy, January 5-10, 2004. Boca Raton, FL: Chapman \& Hall/CRC. Lecture Notes in Pure and Applied Mathematics, 245, pp. 133-148, 2004. 
[11] Gozzi F., Marinelli C., Savin S., On controlled linear diffusions with delay in a model of optimal advertising under uncertainty with memory effects, Journal of Optimization, Theory and Applications, to appear.

[12] Larssen B., Dynamic programming in stochastic control of systems with delay, Stochastics and Stochastics Reports, 74, 3-4, pp 651-673, 2002.

[13] Larssen B., Risebro N.H., When are HJB-equations for control problems with stochastic delay equations finite-dimensional?, Stochastic Analysis and Applications, 21, 3, pp. 643671, 2003.

[14] Mordecki E., Optimal stopping and perpetual options for Lévy processes, Finance and Stochastics, 6, pp. 473-493, 2002.

[15] Øksendal B., Sulem A., A maximum principle for optimal control of stochastic systems with delay with applications to finance, Optimal Control and PDE, Essays in Honour of Alain Bensoussan, eds J.L. Menaldi, E. Rofman and A. Sulem, IOS Press, Amsterdam, pp. 64-79, 2001.

[16] Øksendal B., Sulem A., Applied Stochastic Control of Jump Diffusions. Second Edition, Springer, 2007.

[17] Øksendal B., Zhang T., Optimal control with partial information for stochastic Volterra equations, Preprint of University of Oslo, 2008.

[18] Peskir G., Shirayev A., Optimal Stopping and Free-Boundary Problems, Birkhauser, 2006.

[19] Peszat S., Zabczyk J., Stochastic partial differential equtions with Lévy noise, Encyclopedia of Mathematics and its Applications, Vol. 113, Cambridge University Press, Cambridge (UK), 2008.

[20] Protter P.E., Stochastic Integration and Differential Equations, 2nd edition, SpringerVerlag, Berlin-Heidelberg-New York, 2003.

[21] Reiss M., Riedle M., Van Gaans O., Delay differential equations driven by Lévy processes: stationarity and Feller's properties, Stochastic processes and their applications, Vol.116, pp. 1409-1432, 2006.

[22] Reiss M., Riedle M., Van Gaans O., On Émery's inequality and a variation-of-constants formula, Stochastic Analysis and Applications, Vol. 25, pp. 353-379, 2007.

[23] Vinter R.B., Kwong R.H., The infinite time quadratic control problem for linear systems with state and control delays: an evolution equation approach, SIAM Journal of Control and Optimization, 19, 1, pp. 139-153, 1981. 